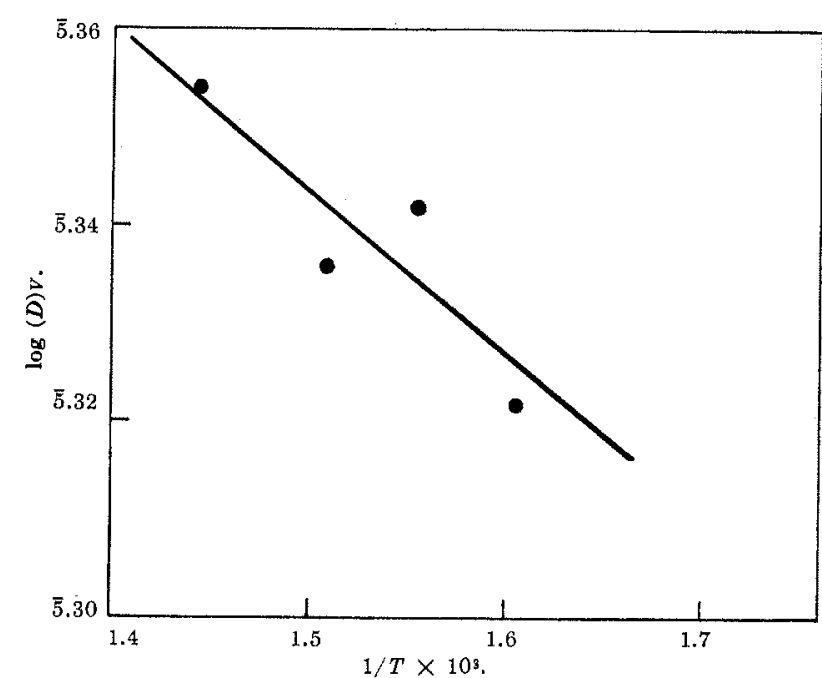

Figure 2. $\log (D)_{V}$ against $1 / T\left({ }^{\circ} \mathrm{K}\right)$.

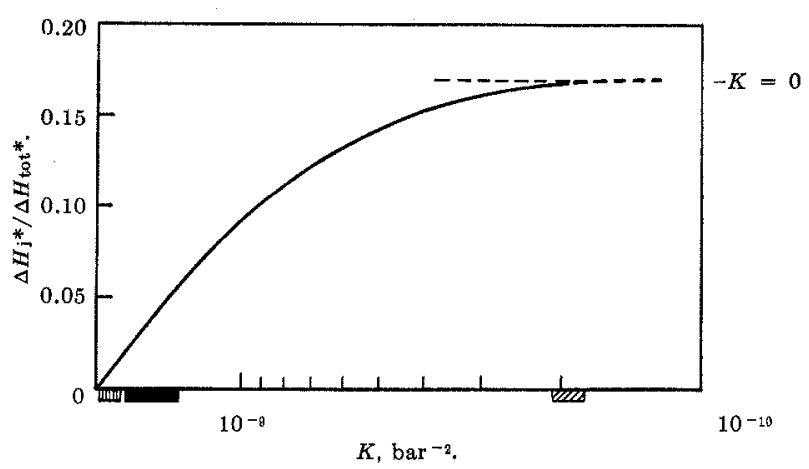

Figure 3. Variation of $\Delta H_{\mathrm{j}}{ }^{*} / \Delta H_{\mathrm{tot}}{ }^{*}$ with

different pressure coefficients $(K)$ of isothermal compressibility $\left[\beta_{P}=\beta_{1}-K P\right]:$ IIIII, alcohols; $\mathbf{\square}$, hydrocarbons; glycols.

The pressures for the constant volume conditions, used in the present calculations, were obtained for the case $K=0$. In the absence of data on the pressure dependence of compressibility of molten salts, only crude estimates of $K$ are possible on the basis of available data $^{13}$ for normal liquids. Figure 3 represents how $\Delta H_{\mathrm{j}}{ }^{*} / \Delta H_{\mathrm{tot}}{ }^{*}$ will change for various values of $K$, estimated from Bridgman's data ${ }^{13}$ on different types of liquids. The classes of liquid are indicated in Fig. 3. It is obvious from Fig. 3 that our value of $\Delta H_{\mathrm{j}}{ }^{*} / \Delta H_{\text {tot }}{ }^{*}$ $\approx 1 / 6$ represents the maximum value for this ratio, consistent with a hole model for molten salts.

The "activation volume", $\Delta V^{*}=-R T(\partial \ln D /$ $\partial P)_{T}$, reported earlier by Tricklebank, Nanis, and Bockris, ${ }^{9}$ i.e., $10.7 \pm 1.4 \mathrm{~cm}^{3}$ mole $^{-1}$, for molten sodium nitrate is found to be constant (within experimental error) in the temperature range studied, i.e., $350-420^{\circ}$.
Acknowledgments. Financial support of the U. S. Atomic Energy Commission under Contract No. AT 30-1-1769 is gratefully acknowledged. The authors are also grateful to Mr. S. R. Richards for many helpful discussions.

(13) P. W. Bridgman, "The Physics of High Pressure," G. Bell and Sons, Ltd., London, 1958.

\section{Stokes' Law Correction Factors for Ionic Motion in Aqueous Solution}

by Eduardo J. Passeron

Facultad de Ciencias Exactas y Naturales. Universidad de Buenos Aires, Argentina (Received October 11.1963)

Stokes' law has been used repeatedly to describe quantitatively ionic motion in infinitely dilute solution. Robinson and Stokes ${ }^{1}$ have used measured ionic conductances and estimated ionic radii for the tetraalkylammonium ions to obtain the relation between the nonsolvated radii of these ions and the values which may be calculated from experimental ionic conductances if Stokes' law is considered valid. They are thus able to obtain a correction factor for Stokes' law, defined by

$$
r=\frac{f}{6 \pi u \eta}
$$

where $r$ is the ionic crystal radius of the ion whose absolute mobility is $u, \eta$ is the solvent viscosity, and $f$ is the correction factor.

These authors find that $f$ is a function of the value

$$
r_{\mathrm{s}}=\frac{1}{6 \pi u \eta}
$$

called "Stokes' law radius" of the ion with absolute mobility $u$. For Stokes' law radii greater than $6 \AA$., the value of the correction factor equals unity, but for smaller ions $f$ increases as $r_{s}$ decreases. Actual values are given below.

An explanation of the deviation from Stokes' law has been suggested by Fuoss ${ }^{2}$ and later rigorously developed using dielectric relaxation theory by Boyd ${ }^{3}$ and Zwan-

(1) R. A. Robinson and R. H. Stokes, "Electrolyte Solutions," Butterworth Scientific Publications, London, 1959, p. 125.

(2) R. M. Fuoss, Proc. Natl. Acad. Sci. U. S., 45, 807 (1959).

(3) R. H. Boyd, J, Chem. Phys., 35, 1281 (1961). 
zig. ${ }^{4}$ Their equation correctly predicts the $f$ values to which we are referring.

The purpose of the present work is to show that an alternative mechanism of energy dissipation, not involving dielectric relaxation, may also lead to an explanation of the $f$ values of the alkylammonium ions. Deviations from Stokes' law are considered here as arising through the following sequence of events. Those solvent molecules which are near enough to the moving ion slip around it in an oriented position, thus rotating in the external electric field. When the ion has passed them, the dipoles revert to their normal position having no preferential orientation. In this way the solvent molecules store up potential energy as they rotate in the external field and later give it up in thermal collisions when the field of the receding ion is no longer sufficiently strong to hold them in the adequate orientation.

The total work $W$ done by the electric field in moving an ion through unit distance is then

$$
W=E^{\prime} e_{0}+w_{\mathrm{rot}}\left(n_{\mathrm{H}_{2} \mathrm{O}} / x\right)
$$

where $w_{\text {rot }}$ is the work done by a $180^{\circ}$ rotation of a water molecule in the external field $E^{\prime}, n_{\mathrm{H}_{2} \mathrm{O}}$ is the number of water molecules undergoing this rotation, $x$ is the distance through which the ion has moved, and $e_{0}$ is the ionic charge. Should Stokes' law apply, the same value of $W$ could be obtained as the work necessary to move an ion of greater radius $r_{\mathrm{eq}}$ through the same distance, $r_{\mathrm{eq}}$ being given by

$$
r_{\mathrm{eq}}=\frac{W}{6 \pi \eta v}=\frac{F W}{6 \pi E^{\prime} \eta \lambda^{0}}
$$

where $F$ is the Faraday and $\lambda^{0}$ is the ionic conductance at infinite dilution. The quotient $r_{\mathrm{eq}} / r_{\mathrm{s}}$ can now be used as a correction factor for Stokes' law and its value equated to the corresponding $f$ value of the same alkylammonium ion. The expression for $f$ would then be

$$
\frac{r_{\mathrm{eq}}}{r_{\mathrm{s}}}=\frac{W}{E^{\prime} e_{0}}=1+\frac{w_{\mathrm{rot}} n_{\mathrm{H}_{2} \mathrm{O}}}{E^{\prime} e_{0}}=f
$$

In this model $n_{\mathrm{H}_{2} \mathrm{O}}$ is approximately given by

$$
{ }^{4} / 3 \pi \frac{\rho^{3}-r_{\mathrm{c}}^{3}}{\rho v_{\mathrm{H}_{2} \mathrm{O}}}
$$

where $v_{\mathrm{H}_{2} \mathrm{O}}$ is the apparent molecular volume of water in the vicinity of the ion, $r_{\mathrm{c}}$ is the crystalline radius, and $\rho$ is the distance from the ion's center up to which all dipoles are considered completely oriented. The value of $\rho$ depends on the equation or equations used to $\mathrm{ex}$ press the field intensity. The ion is considered here as a rigid sphere of radius $r_{\mathrm{c}}$ with the ionic charge $e_{0}$ located at its geometrical center. It is assumed that the electrie field within this sphere varies according to Coulomb's law

$$
E=\frac{e_{0}}{\epsilon r^{2}}
$$

where the dielectric constant $\epsilon$ is taken as equal to 1.8 on the basis of the known values for static dielectric constants of liquid saturated $n$-hydrocarbons. Outside the ion's sphere, the electric field is assumed to vary according to the equation

$$
n^{2} E+\left[\left(\epsilon_{0}-n^{2}\right) / B^{1 / 2}\right] t g^{-1} B^{1 / 2} E=\frac{e_{0}}{r^{2}}
$$

Here $\epsilon_{0}$ is the static dielectric constant of the solvent, $n$ is the refractive index, and $B$ is a constant which can be calculated theoretically. This equation can be obtained from Booth's $s^{5}$ theory of the differential dielectric constant as shown by Laidler. ${ }^{6}$

$\rho$ is now expressed by the equation

$$
\rho=r_{\mathrm{o}}+r_{\rho}-r^{\prime}
$$

with $r_{\rho}$ an adjustable parameter and $r^{\prime}$ the value of the distance in eq. 5 at which $E$ has a common value in eq. 5 and in eq. $4, r_{\mathrm{c}}$ being introduced in the latter equation.

For comparison with experimental data, $f$ can be written as

$$
f=1+b \frac{\rho^{3}-r_{\mathrm{c}}^{3}}{\rho}
$$

\begin{tabular}{|c|c|c|c|c|}
\hline & $f_{\text {exptl }}$ & $f_{\text {oaled }}$ & $\AA^{b-2}$ & $\begin{array}{l}\gamma_{\rho}, \\
\AA . \\
\AA\end{array}$ \\
\hline $\mathrm{N}\left(\mathrm{CH}_{3}\right)_{4}+$ & 1.70 & 1.70 (fit) & 0.37 & 1.96 \\
\hline $\mathrm{N}\left(\mathrm{C}_{2} \mathrm{H}_{5}\right)_{4}+$ & 1.42 & 1.49 & & \\
\hline $\mathrm{N}\left(\mathrm{C}_{8} \mathrm{H}_{7}\right)_{4}+$ & 1.15 & 1.30 & & \\
\hline $\mathrm{N}\left(\mathrm{C}_{4} \mathrm{H}_{9}\right)_{4}+$ & 1.05 & 1.15 & & \\
\hline $\mathrm{N}\left(\mathrm{C}_{5} \mathrm{H}_{11}\right)_{4}{ }^{+*}$ & 1.01 & 1.00 & & \\
\hline
\end{tabular}

where $b$ is a second adjustable parameter. If this equation is fitted to the values of $f$ by choosing $b$ so that the calculated and the experimental values of $f$ for methylammonium ion coincide, the values in Table I are obtained.

\section{Table I}

Although the values of $b$ and $r_{\rho}$ are determined by the best fit to experimental data, the interpretation of $f$ given here assigns physical significance to each of them.

(4) R. Zwanzig, J. Chem. Phys., 38, 1603 (1963)

(5) F. Booth, ibid., 19, 391, 1327, 1615 (1951).

(6) K. J. Laidler, Can. J. Chem., 37, 138 (1959). 
$b$ is related to the dipole moment of the water molecule, the angle $\theta$ through which it rotates as it passes near the ion, and the volume it occupies, as shown by the equation

$$
b=4 / 3 \pi \mu \frac{\cos \theta}{e_{0} v_{\mathrm{H}_{2} \mathrm{O}}}
$$

If every water molecule between $r_{\mathrm{c}}$ and $\rho$ is imagined undergoing a complete $180^{\circ}$ rotation in the external field and the value of the permanent dipole moment of the gaseous water molecule is taken for $\mu$, then the volume $v_{\mathrm{H}_{2}} \mathrm{O}$ required by the best fit value of $b$ is $8 \AA^{3}$. Although this is considerably less than the apparent molecular volume of water in the bulk $\left(30 \AA .{ }^{3}\right)$, it may not be physically unreasonable considering the $\mathrm{O}-\mathrm{H}$ distance of $0.97 \AA$. in the water molecule and the packing of solvent molecules near an ion.

According to theory $\rho$ is that distance measured from the center of the ion at which the complete orientation of water molecules in the ion's field is imagined to cease abruptly. This is of course an over-simplified picture. The average angle that the dipole moment vector forms with the radial vector varies with the distance, from approximately 0 at the surface of the ion to $\pi / 2$ far from the ion where thermal movement predominates. Tevertheless, it seems reasonable to expect the rotational effect described here to become unimportant when the value of the ion-dipole interaction becomes approximately equal to average thermal energy. The best fit value of $r_{\rho}$ corresponds to an $E_{\mu} / k T$ value of approximately unity in agreement with the argument above.

Acknowledgments. The author wishes to thank Dr. Roger Parsons of Bristol University and Dr. Félix Cernuschi and Dr. Antonio Misetich of the University of Buenos Aires for helpful discussion of some aspects of this work.

\section{The Critical Surface Tension of Glass}

\author{
by Douglas A. Olsen and A. Jean Osteraas \\ T. L. Daniels Research Center, Archer-Daniels-Midland Company, \\ Minneapolis 20, Minnesota (Received February 12, 1964)
}

Zisman, et al., ${ }^{1,2}$ have shown that the critical surface tension, $\gamma_{\mathrm{c}}$, can be obtained for low surface energy materials. In later studies ${ }^{3,4}$ on high energy surfaces such as $\mathrm{SiO}_{2}$, the organic liquids which were used either spread spontaneously or were autophobic.
It is a well-known fact that an $\mathrm{SiO}_{2}$ (or glass) surface may adsorb a considerable amount of water giving rise to many phenomena. ${ }^{.}$This fact prompted this investigation since it seemed probable that the adsorbed water layer on glass surfaces would result in relatively low critical surface tension values.

\section{Experimental Procedure}

Potassium carbonate and calcium chloride solutions were chosen since their surface tensions exceed that of water. $^{6}$ The solutions were prepared using analytica] grade reagents and distilled water. The reported values $^{6}$ for the surface tensions of the salt solutions were verified using sessile drop measurements on a Teflon substrate.

The absence of organic contaminants in the distilled water was demonstrated by evaporating the water from the chemically cleaned glass surfaces. Following evaporation, zero contact angles were obtained with additional water on the affected areas thus indicating the absence of organic contaminants.

The glass surfaces were cleaned by the following sequence: (1) a detergent wash, (2) a distilled water rinse, (3) a hot concentrated nitric acid wash, (4) copious rinsing in distilled water, and (5) drying in a desiccator. All the glass surfaces used exhibited zero contact angles with distilled water.

Most of the contact angle measurements were made after a short drying period. No detectable differences in contact angles were observed for longer drying periods. The drops of salt solution were placed on the glass surface and additional solution was added until a constant advancing contact angle was obtained. The contact angles were measured to within $\pm 2^{\circ}$ using an Eberbach telescopic eyepiece. All measurements were made at $20^{\circ}$ and $20 \%$ relative humidity.

\section{Results and Discussion}

The results of contact angle measurements are shown in Fig. 1A and suggest a critical surface tension, $\gamma_{c}$, of 73 dynes $/ \mathrm{cm}$., which is approximately that of water at $20^{\circ}$. The curvature probably results because of hydrogen bonds formed between the water molecules in solution and those adsorbed on the glass surface. ${ }^{7}$ The results

(1) H. W. Fox and W. A. Zisman, J. Colloid Sci., 5, 514 (1950); 7, $109,428(1952)$.

(2) M. K. Bernett and W. A. Zisman, J. Phys. Chem., 63, 1241 (1959).

(3) E. F. Hare and W. A. Zisman, ibid., 59, 335 (1955).

(4) H. W. Fox, E. F. Hare, and W. A. Zisman, ibid., 59, 1097 (1955). (5) W, Eitel, "The Physical Chemistry of the Silicates," University of Chicago Press, Chicago, Ill., 1954, p. $1338 \mathrm{ff}$.

(6) N. A. Lange, "Handbook of Chemistry," 10th Ed., McGraw-Hill Book Co., Inc., New York, N. Y., 1961, p. 1652. 